\title{
Microscopic theory of optical absorption in graphene enhanced by lattices of plasmonic nanoparticles
}

\author{
Niclas S. Mueller and Stephanie Reich \\ Department of Physics, Freie Universität Berlin, Arnimallee 14, D-14195 Berlin, Germany
}

(Received 3 April 2018; revised manuscript received 22 May 2018; published 11 June 2018)

\begin{abstract}
We present a microscopic description of plasmon-enhanced optical absorption in graphene, which is based on perturbation theory. We consider the interaction of graphene with a lattice of plasmonic nanoparticles, as was previously realized experimentally. By using tight-binding wave functions for the electronic states of graphene and the dipole approximation for the plasmon, we obtain analytic expressions for the coupling matrix element and enhanced optical absorption. The plasmonic nanostructure induces nonvertical optical transitions in the band structure of graphene with selection rules for the momentum transfer that depend on the periodicity of the plasmonic lattice. The plasmon-mediated optical absorption leads to an anisotropic carrier population around the $K$ point in phase space, which depends on the polarization pattern of the plasmonic near field in the graphene plane. Using Fourier optics, we draw a connection to a macroscopic approach, which is independent from graphene-specific parameters. Each Fourier component of the plasmonic near field corresponds to the momentum transfer of an optical transition. Both approaches lead to the same expression for the integrated optical absorption enhancement, which is relevant for the photocurrent enhancement in graphene-based optoelectronic devices.
\end{abstract}

DOI: 10.1103/PhysRevB.97.235417

\section{INTRODUCTION}

Graphene possesses extraordinary optical properties, such as a constant optical absorption over a broad spectral range, a strongly nonlinear optical response, and tunable intrinsic plasmons [1,2]. The optical response is efficiently tuned by gating, doping, and chemical functionalization, which makes graphene an attractive material for broadband photodetection and ultrafast light modulation [3,4]. Although graphene's optical absorption and quantum efficiency are exceptionally high (2.3\% per monolayer) for a single layer of atoms [5,6], it remains too low for building photonic devices.

The linear optical properties of graphene are well described by microscopic formalisms that are based on a tight-binding approximation for the electronic states [7-10]. The microscopic approaches give insight into the distribution and dynamics of photoexcited carriers and are key for understanding the doping and excitation-energy dependence of graphene's optical absorption and Raman response [9,11,12]. The linear and gapless energy dispersion of graphene close to the Dirac points gives rise to a number of fascinating optical phenomena. An anisotropic population of photogenerated carriers was predicted around the $K$ point as a manifestation of the sublattice pseudospin $[9,13]$. The anisotropy and ultrafast carrier relaxation was measured in polarization-resolved pump-probe experiments, which showed good agreement with the microscopic calculations [14-19]. Furthermore, it might explain a dependence of the photocurrent in graphene-based photodetectors on the light polarization [20].

The optical response of graphene may be modified and tailored by a combination with other materials [21,22]. It was proposed that molecular dipole fields on functionalized graphene induce indirect optical transitions with a momentum transfer proportional to the molecular density [23]. This effect could be exploited for the activation of intrinsically dark optical processes, such as excitons in transition metal dichalcogenides [24]. A promising approach to enhance the optical response of graphene is the coupling to electromagnetic near fields generated by localized surface plasmon resonances (LSPR) in noble metal nanostructures [22]. It was experimentally demonstrated that a lattice of gold nanoparticles deposited on top or below graphene can enhance the quantum efficiency in photodetectors as well as the Raman response by more than one order of magnitude [25-28]. The exact mechanism of photocurrent generation remains a matter of debate. Hot electron injection and thermoelectric effects were proposed to act additionally to the electromagnetic field enhancement [20,28-30]. The enhancement of optical processes in graphene by the local near fields of localized surface plasmons was so far only described by a purely electromagnetic theory [25-28]; a microscopic approach is missing.

Here we present a microscopic theory of optical transitions in graphene that are induced by the coupling to localized surface plasmon resonances in metallic nanostructures. Our theoretical framework is based on perturbation theory and a tight-binding model for the electronic states of graphene. We consider a lattice of plasmonic nanoparticles that couples to graphene via its electric near field. To obtain a qualitative description of the interaction, we derive analytic expressions for the interband transition amplitudes and the optical absorption enhancement. The plasmonic nanostructure induces nonvertical interband transitions in graphene with momentum transfers that depend on the periodicity of the plasmonic lattice. We show that each momentum transfer corresponds to the spatial frequency of one Fourier component of the plasmonic near field. The distribution of photoexcited carriers in phase space depends on the polarization pattern of the electric field in the graphene plane. The optical 
absorption enhancement that is relevant for the photocurrent in optoelectronic devices is independent of graphene-specific parameters and identical with the enhancement obtained from a macroscopic approach.

The paper is organized as follows. In Sec. II we present the theoretical framework that is used throughout the paper. In Sec. III we derive the plasmon-electron matrix element. In Sec. IV we calculate the distribution of photoexcited carriers in phase space and compare plasmon-mediated optical transitions with transitions by direct absorption of the incident light. In Sec. V we calculate the integrated optical absorption, which is, e.g., relevant for the photocurrent enhancement, and draw a comparison to a macroscopic approach. Finally, in Sec. VI we estimate how a generalization beyond the quasistatic approximation affects the main conclusions of the paper. Section VII contains the conclusions.

\section{METHODS}

\section{A. Optical response of the nanoparticle lattice}

Following past experiments [25-27], we consider a square lattice of plasmonic particles, where the spacing $\Delta R$ between the particles is larger than half of the lateral particle size (schematically depicted in Fig. 1). We use the quasistatic approximation and dipole approximation for the electric potential generated by the plasmonic lattice

$$
\phi_{\mathrm{pl}}(\mathbf{r})=\sum_{\mathbf{R}_{\mathrm{pl}}} \frac{\mathbf{p} \cdot\left(\mathbf{r}-\mathbf{R}_{\mathrm{pl}}\right)}{4 \pi \epsilon_{0}\left|\mathbf{r}-\mathbf{R}_{\mathrm{pl}}\right|^{3}}
$$

to obtain an analytic expression for the interaction matrix element. The sum is carried out over the positions $\mathbf{R}_{\mathrm{pl}}$ of each plasmonic nanoparticle. $\mathbf{p}=4 \pi \epsilon_{0} \alpha_{\mathrm{pl}}(\omega) E_{0} \boldsymbol{\varepsilon}_{\mathrm{pt}}$ is the dipole moment induced in each particle by an external light field with amplitude $E_{0}$, frequency $\omega$, and polarization $\boldsymbol{\varepsilon}_{\mathrm{pt}}$. $\alpha_{\mathrm{pl}}(\omega)$ is the polarizability of a nanoparticle in the plasmonic lattice; see below. The dipole approximation is strictly only valid for spherical particles that are much smaller than the wavelength of the incoming light. We therefore abstain from a quantitative comparison to experiments in this work and focus on a qualitative understanding of the interaction between the plasmonic lattice and graphene. In the following we assume that the plasmonic dipoles are placed in the $x y$ plane at $z=R_{z}$ and the graphene sheet at $z=0$. Furthermore, we consider

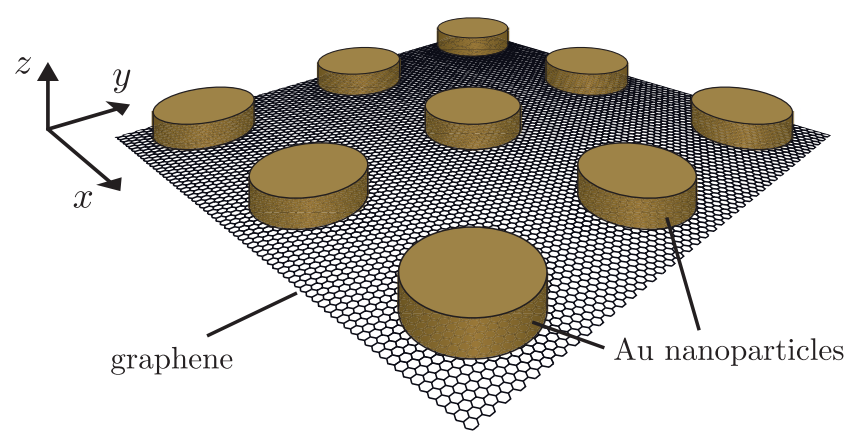

FIG. 1. Sketch of the experimental system that is considered in this work. A rectangular lattice of gold nanoparticles is deposited on graphene. incident light that propagates perpendicular to the $x y$ plane, i.e., $\boldsymbol{\varepsilon}_{\mathrm{pt}}=\left\{\varepsilon_{x}^{\mathrm{pt}}, \varepsilon_{y}^{\mathrm{pt}}\right\}$.

To calculate the polarizability $\alpha_{\mathrm{pl}}(\omega)$ of the nanoparticles, we use an analytical model for the dielectric function of gold $\epsilon_{\mathrm{Au}}(\omega)$ from Ref. [31] to reproduce the experimental data from Ref. [32]. We assume that the gold nanoparticles have the shape of oblate spheroids, with the radii $r_{x}=r_{y}=: r_{x y}$ and $r_{z}<r_{x y}$ (see inset of Fig. 2). The optical polarizability of a single nanoparticle is calculated as [33]

$$
\alpha_{\mathrm{pl}}^{\mathrm{os}}\left(\omega, r_{x y}, r_{z}\right)=\frac{r_{x y}^{2} r_{z}}{3} \frac{\epsilon_{\mathrm{Au}}(\omega)-\epsilon_{\mathrm{m}}}{L_{x y} \epsilon_{\mathrm{Au}}(\omega)+\epsilon_{\mathrm{m}}\left(1-L_{x y}\right)},
$$

with

$$
L_{x y}=\frac{1}{2 e_{0}^{2}}\left(\frac{\sqrt{1-e_{0}^{2}}}{e_{0}} \arcsin e_{0}-1+e_{0}^{2}\right)
$$

and

$$
e_{0}=1-\frac{r_{z}^{2}}{r_{x y}^{2}}
$$

$\epsilon_{\mathrm{m}}$ is the dielectric constant of the background medium. We consider $\epsilon_{\mathrm{m}}=1$ in this work. We neglect the coupling between the nanoparticles, as near field interactions or lattice resonances lead only to negligible shifts for the gap sizes considered in this work [34]. The lattice of Au nanoparticles that is considered in this work has a localized-surface plasmon resonance at $2.3 \mathrm{eV}$; see Fig. 2.

\section{B. Tight-binding wave functions of graphene}

We use a tight-binding model for the electronic wave functions of graphene that is suitable for obtaining analytic expressions for the optical response of graphene at visible light frequencies [7-10]. The wave functions are written as a linear combination of Bloch sums for the two sublattices $A$ and

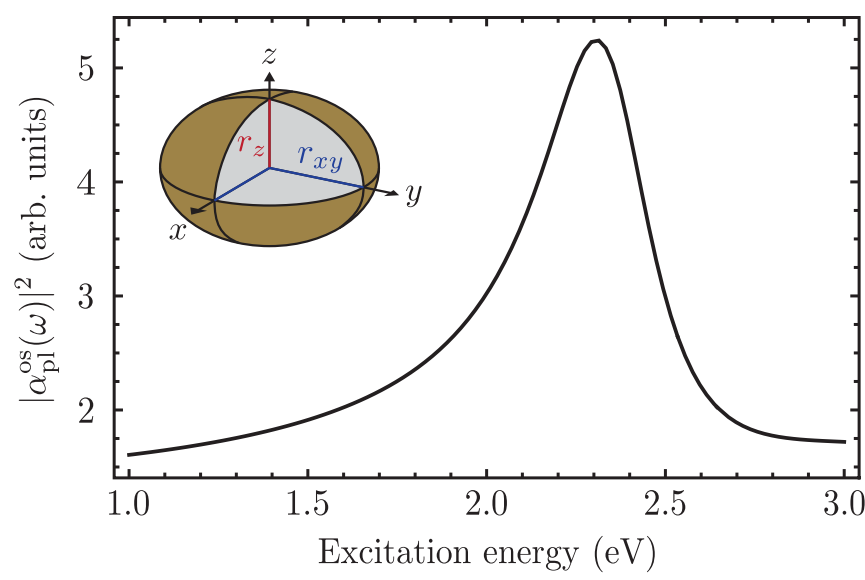

FIG. 2. Plot of the polarizability $\left|\alpha_{\mathrm{pl}}^{\mathrm{os}}(\omega)\right|^{2}$ of a nanoparticle in the plasmonic lattice with an optical resonance at $2.3 \mathrm{eV}$. The nanoparticle is modeled as an oblate Au spheroid with $r_{x y}=20 \mathrm{~nm}$ and $r_{z}=10 \mathrm{~nm}$; see inset. 
$B$ [35]:

$$
\left|\Psi^{\beta}(\mathbf{k}, \mathbf{r})\right\rangle=\sum_{j=A, B} C_{j}^{\mathrm{v}}(\mathbf{k})\left|\Phi_{j}(\mathbf{k}, \mathbf{r})\right\rangle,
$$

with the band index $\beta=\mathrm{v}, \mathrm{c}$. The Bloch sums

$$
\left|\Phi_{j}(\mathbf{k}, \mathbf{r})\right\rangle=\frac{1}{\sqrt{N_{\mathrm{c}}}} \sum_{\mathbf{R}_{j}} e^{i \mathbf{k} \cdot \mathbf{R}_{j}}\left|\psi_{p z}\left(\mathbf{r}-\mathbf{R}_{j}\right)\right\rangle
$$

are calculated over a finite number of $N_{\mathrm{c}}$ unit cells of the graphene sublattices $j=A, B$ with atomic positions $\mathbf{R}_{j}$. We use a Gaussian basis set from Ref. [36] for the atomic carbon $2 p_{z}$ orbitals $\psi_{p z}(\mathbf{r})$ that is fit into ab initio data from Ref. [37]; see the Supplemental Material [38]. We use the wave-function coefficients [36]

$$
C_{A}^{\mathrm{v}}(\mathbf{k})=C_{A}^{\mathrm{c}}(\mathbf{k})=\sqrt{\frac{\alpha(\mathbf{k})}{2|\alpha(\mathbf{k})|}}
$$

and $C_{B}^{\mathrm{v}}(\mathbf{k})=-C_{B}^{\mathrm{c}}(\mathbf{k})=\left[C_{A}^{\mathrm{v}}(\mathbf{k})\right]^{*}$, where

$$
\alpha(\mathbf{k})=\sum_{j=1}^{3} e^{i \mathbf{k} \cdot \delta_{j}},
$$

with the nearest-neighbor vectors $\boldsymbol{\delta}_{i}$. We neglected the nearestneighbor overlap $s_{0}$.

Close to the $K$ points $\mathbf{K}_{+}=4 \pi /\left(3 \sqrt{3} a_{\mathrm{cc}}\right)\{\sqrt{3} / 2,1 / 2\}$ and $\mathbf{K}_{-}=4 \pi /\left(3 \sqrt{3} a_{\mathrm{cc}}\right)\{\sqrt{3} / 2,-1 / 2\}$ in the Brillouin zone of graphene, the energy dispersion can be approximated as a cone $\epsilon_{\mathrm{el}}^{\mathrm{c}}\left(\mathbf{k}_{ \pm}\right)=\hbar v_{\mathrm{F}}\left|\mathbf{k}_{ \pm}\right|$and $\epsilon_{\mathrm{el}}^{\mathrm{v}}\left(\mathbf{k}_{ \pm}\right)=-\hbar v_{\mathrm{F}}\left|\mathbf{k}_{ \pm}\right|$, where $\mathbf{k}_{ \pm}=\mathbf{k}-$ $\mathbf{K}_{ \pm}$(linear-band approximation). $v_{\mathrm{F}}=3 a_{\mathrm{cc}} \gamma_{0} / 2 \hbar \approx 8.5 \times$ $10^{5} \mathrm{~m} / \mathrm{s}$ is the Fermi velocity, with the nearest-neighbor hopping parameter $\gamma_{0} \approx 2.6 \mathrm{eV}$ and the carbon-carbon distance $a_{\mathrm{cc}}=1.42 \AA$ [39]. The wave-function coefficients are simplified with the approximation $\alpha\left(\mathbf{k}_{ \pm}\right) \approx 3 a_{\mathrm{cc}}\left(i k_{x}^{ \pm} \pm k_{y}^{ \pm}\right) / 2$.

\section{INTERACTION MATRIX ELEMENTS}

We first derive an analytic expression for the plasmonelectron matrix element, which is used later to calculate the transition probability and enhanced optical absorption of graphene. The gauge independent single-electron interaction Hamiltonian is [40]

$$
\begin{aligned}
\mathcal{H}_{\mathrm{el}-\mathrm{pl}}(\mathbf{r})= & -\frac{i \hbar e}{4 m}\left[\nabla_{\mathbf{r}} \cdot \mathbf{A}_{\mathrm{pl}}(\mathbf{r})+2 \mathbf{A}_{\mathrm{pl}}(\mathbf{r}) \cdot \nabla_{\mathbf{r}}\right] \\
& -\frac{e}{2} \phi_{\mathrm{pl}}(\mathbf{r})+O\left(\mathbf{A}_{\mathrm{pl}}^{2}\right),
\end{aligned}
$$

with the vector potential $\mathbf{A}_{\mathrm{pl}}(\mathbf{r})$ and scalar potential $\phi_{\mathrm{pl}}(\mathbf{r})$ of the plasmonic nanostructure. We assume time-harmonic fields and used the rotating-wave approximation. Within the quasistatic approximation, i.e., considering that the size of each plasmonic particle is much smaller than the wavelength of light, the interaction Hamiltonian reduces to the electrostatic potential

$$
\mathcal{H}_{\mathrm{el-pl}}(\mathbf{r}) \approx-\frac{e}{2} \phi_{\mathrm{pl}}(\mathbf{r})
$$

The matrix element for a plasmon-induced optical transition from an electronic state with wave vector $\mathbf{k}_{\mathrm{v}}$ in the valence band to a state $\mathbf{k}_{\mathrm{c}}$ in the conduction band is

$$
\mathcal{M}_{\mathrm{el}-\mathrm{pl}}^{\mathbf{k}_{\mathrm{c}}, \mathbf{k}_{\mathrm{v}}}=-\frac{e}{2}\left\langle\Psi^{\mathrm{c}}\left(\mathbf{k}_{\mathrm{c}}, \mathbf{r}\right)\left|\phi_{\mathrm{pl}}(\mathbf{r})\right| \Psi^{\mathrm{v}}\left(\mathbf{k}_{\mathrm{v}}, \mathbf{r}\right)\right\rangle
$$

Considering only 0th-neighbor interactions, we obtain

$$
\begin{aligned}
\mathcal{M}_{\mathrm{el}-\mathrm{pl}}^{\mathbf{k}_{\mathrm{c}}, \mathbf{k}_{\mathrm{v}}}= & -\frac{e}{2 N_{\mathrm{c}}} \sum_{j=\mathrm{A}, \mathrm{B}}\left(C_{j}^{\mathrm{c}}\left(\mathbf{k}_{\mathrm{c}}\right)\right)^{*} C_{j}^{\mathrm{v}}\left(\mathbf{k}_{\mathrm{v}}\right) \\
& \times \sum_{\mathbf{R}_{j}} e^{i\left(\mathbf{k}_{\mathrm{v}}-\mathbf{k}_{\mathrm{c}}\right) \cdot \mathbf{R}_{j}} \phi_{\mathrm{pl}}\left(\mathbf{R}_{j}\right) .
\end{aligned}
$$

We approximated

$$
\left\langle\psi_{p z}\left(\mathbf{r}-\mathbf{R}_{j}\right)\left|\phi_{\mathrm{pl}}(\mathbf{r})\right| \psi_{p z}\left(\mathbf{r}-\mathbf{R}_{j}\right)\right\rangle \approx \phi_{\mathrm{pl}}\left(\mathbf{R}_{j}\right),
$$

because the potential of the plasmonic nanostructure $\phi_{\mathrm{pl}}(\mathbf{r})$ may be considered constant over the volume of the carbon $p_{z}$ orbital. We also confirm that first-nearest-neighbor contributions to $\mathcal{M}_{\mathrm{el}-\mathrm{pl}}$ are negligible within the quasistatic approximation [38].

One might proceed here by directly calculating the lattice sums that appear in Eq. (12). This would require the choice of a unit cell, which leads to an expression similar to the dipole matrix element $-e \mathbf{r} \cdot \mathbf{E}$ that describes the interaction of light with molecules [38]. It was shown by Binder and co-workers that this matrix element cannot be applied for Bloch wave functions with periodic boundary conditions $[41,42]$. We therefore proceed by calculating the matrix element in Fourier space, as was done for the interaction of molecular dipole potentials with graphene [23].

Because of its periodicity, the electric potential of the plasmonic nanostructure can be expressed as a Fourier sum [38]

$$
\phi_{\mathrm{pl}}(\mathbf{r})=-\frac{i \mathcal{N}_{\mathrm{pl}}}{2 \epsilon_{0}} \sum_{\Delta \mathbf{q} \neq 0} \frac{\mathbf{p} \cdot \Delta \mathbf{q}}{|\Delta \mathbf{q}|} e^{i \Delta q_{x} x} e^{i \Delta q_{y} y} e^{-|\Delta \mathbf{q}|\left|z-R_{z}\right|},
$$

where $\mathcal{N}_{\mathrm{pl}}$ is the density of the plasmonic nanoparticles. Each of the Fourier components oscillates with a spatial frequency $\Delta q_{x}=2 \pi m_{x} / \Delta R$ and $\Delta q_{y}=2 \pi m_{y} / \Delta R$ in the $x y$ plane, where $m_{x}, m_{y} \in \mathbb{Z}$ and $\Delta \mathbf{q}=\left\{\Delta q_{x}, \Delta q_{y}\right\}$ (see Fig. 3). The Fourier components drop exponentially with the magnitude of the spatial frequency $|\Delta \mathbf{q}|$ and the distance $\left|z-R_{z}\right|$ to the plane of plasmonic dipoles. Using Eq. (14) and the relation

$$
\frac{1}{N_{\mathrm{c}}} \sum_{\mathbf{R}_{j}} e^{i\left[\Delta \mathbf{q}-\left(\mathbf{k}_{\mathrm{c}}-\mathbf{k}_{\mathrm{v}}\right)\right] \cdot \mathbf{R}_{j}}=\delta_{\Delta \mathbf{q}, \mathbf{k}_{\mathrm{c}}-\mathbf{k}_{\mathrm{v}}},
$$

it is straightforward to calculate the lattice sums in Eq. (12). We obtain the analytic expression

$$
\begin{aligned}
\mathcal{M}_{\mathrm{el}-\mathrm{pl}}^{\mathbf{k}_{\mathrm{c}}, \mathbf{k}_{\mathrm{v}}}= & \frac{i e \mathcal{N}_{\mathrm{pl}}}{4 \epsilon_{0}} \sum_{\Delta \mathbf{q} \neq 0} \delta_{\Delta \mathbf{q}, \mathbf{k}_{\mathrm{c}}-\mathbf{k}_{\mathrm{v}}} \sum_{j=A, B}\left(C_{j}^{\mathrm{c}}\left(\mathbf{k}_{\mathrm{c}}\right)\right)^{*} C_{j}^{\mathrm{v}}\left(\mathbf{k}_{\mathrm{v}}\right) \\
& \times \frac{\mathbf{p} \cdot\left(\mathbf{k}_{\mathrm{c}}-\mathbf{k}_{\mathrm{v}}\right)}{\left|\mathbf{k}_{\mathrm{c}}-\mathbf{k}_{\mathrm{v}}\right|} e^{-\left|R_{z}\right|\left|\mathbf{k}_{\mathrm{c}}-\mathbf{k}_{\mathrm{v}}\right|}
\end{aligned}
$$

that is independent from the choice of unit cell in the graphene lattice. The interaction of graphene with the plasmonic nanostructure does not depend on the relative orientation of the two lattices as expected.

Interestingly, the plasmonic lattice imposes selection rules on the optical transitions in graphene. A momentum transfer $\mathbf{k}_{\mathrm{c}}-\mathbf{k}_{\mathrm{v}}$ from the plasmonic nanostructure to the electronic states of graphene leads to nonvertical optical transitions. Because of the periodicity of the square lattice of plasmonic nanoparticles, only momentum transfers that satisfy $k_{x}^{\mathrm{c}}-$ 


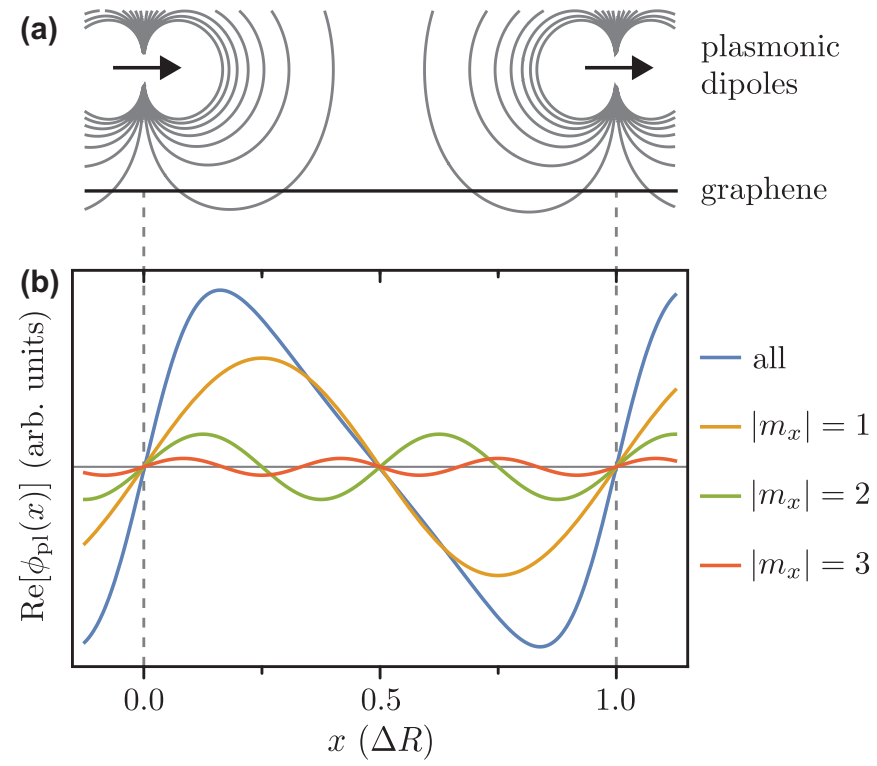

FIG. 3. (a) Sketch of equipotential lines generated by the plasmonic lattice in the $x z$ plane. The plasmonic excitation in each nanoparticle is represented by a point dipole. (b) Plot of the electric potential $\phi_{\mathrm{pl}}$ in the graphene plane along the $x$ axis (blue) and its Fourier components. The Fourier components are calculated as the sum of all components with $\left|m_{x}\right|=1$ (orange), $\left|m_{x}\right|=2$ (green), and $\left|m_{x}\right|=3$ (red). Parameters: $\boldsymbol{\varepsilon}_{\mathrm{pt}}=\mathbf{e}_{x}, \Delta R / R_{z}=4$.

$k_{x}^{\mathrm{v}}=2 \pi m_{x} / \Delta R$ and $k_{y}^{\mathrm{c}}-k_{y}^{\mathrm{v}}=2 \pi m_{y} / \Delta R$ are allowed. The momentum transfer is inversely proportional to the spacing $\Delta R$ between the plasmonic nanoparticles. Each momentum transfer corresponds to the spatial frequency $\Delta \mathbf{q}$ of one Fourier component of the electric potential $\phi_{\mathrm{pl}}(\mathbf{r})$ in Eq. (14). The matrix element vanishes for vertical transitions from valence to conduction band, i.e., $\mathbf{k}_{\mathrm{c}}=\mathbf{k}_{\mathrm{v}}$, because there is no Fourier component of $\phi_{\mathrm{pl}}(\mathbf{r})$ with $\Delta \mathbf{q}=0$. Our theory can be also applied to plasmonic lattices with other periodicity than the square lattice, which would lead to different selection rules for the momentum transfer.

To draw a comparison, we also calculate the matrix element for photon-electron interaction without intermediate excitation of a localized-surface plasmon. The interaction Hamiltonian

$$
\mathcal{H}_{\mathrm{el}-\mathrm{pt}}=-\frac{i \hbar e}{2 m} \mathbf{A}_{\mathrm{pt}} \cdot \nabla_{\mathbf{r}}
$$

is obtained from Eq. (9) using the Coulomb gauge $\nabla_{\mathbf{r}} \cdot \mathbf{A}_{\mathrm{pt}}=$ 0 . The photon-electron matrix element

$$
\mathcal{M}_{\mathrm{el}-\mathrm{pt}}^{\mathbf{k}}=-\frac{i \hbar e}{2 m} \mathbf{A}_{\mathrm{pt}} \cdot\left\langle\Psi^{\mathrm{c}}(\mathbf{k}, \mathbf{r})\left|\nabla_{\mathbf{r}}\right| \Psi^{\mathrm{v}}(\mathbf{k}, \mathbf{r})\right\rangle
$$

is calculated with the approximation $\mathbf{k}=\mathbf{k}_{\mathrm{c}}=\mathbf{k}_{\mathrm{v}}$, neglecting the (small) photon momentum that is oriented perpendicular to the graphene lattice. The matrix element vanishes for 0thneighbor interactions and can be explicitly calculated for firstnearest-neighbor interactions as

$$
\mathcal{M}_{\mathrm{el}-\mathrm{pt}}^{\mathbf{k}_{ \pm}}=\mp \frac{3 e \hbar}{4 m \omega} E_{0} \frac{\varepsilon_{x}^{\mathrm{pt}} k_{y}^{ \pm}-\varepsilon_{y}^{\mathrm{pt}} k_{x}^{ \pm}}{\left|\mathbf{k}^{ \pm}\right|} m_{\mathrm{opt}}
$$

within the linear-band approximation, where $m_{\mathrm{opt}} \approx$ $4.66 \mathrm{~nm}^{-1}[13,38]$.
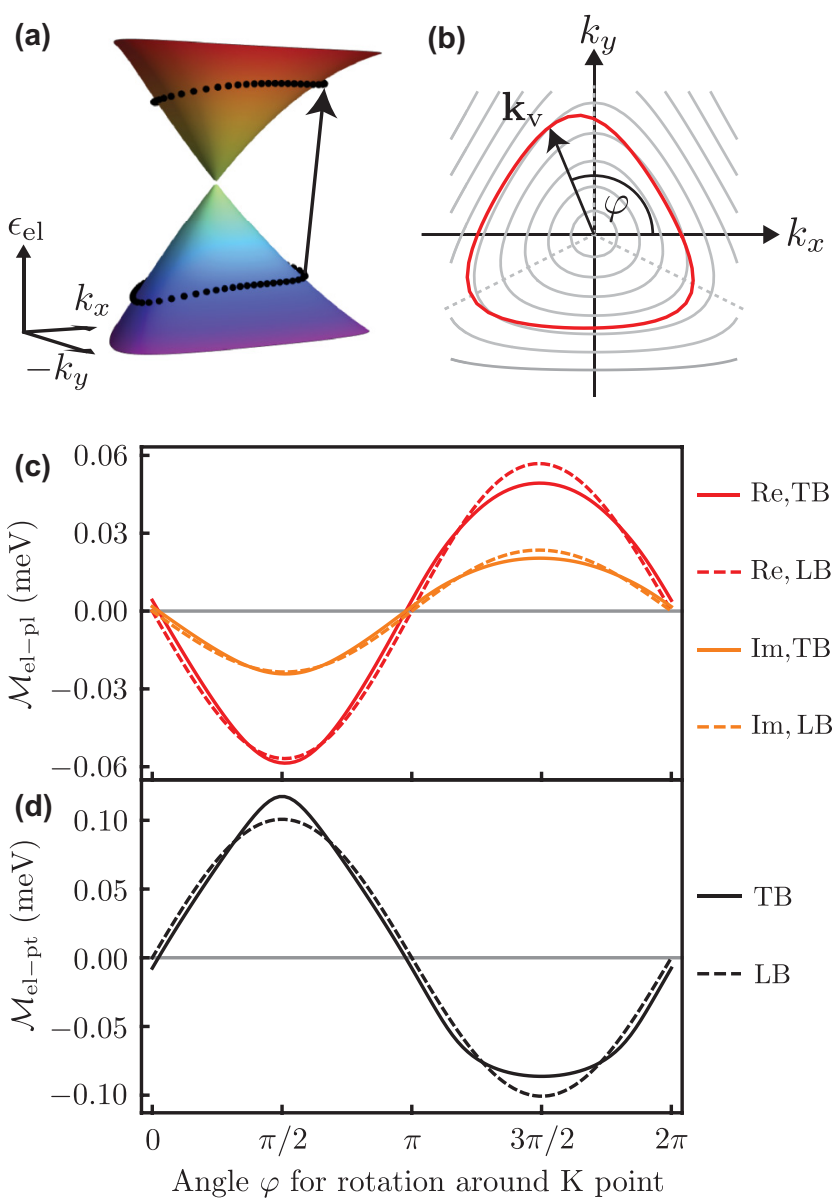

FIG. 4. (a) Sketch of a nonvertical optical transition in the energy dispersion of graphene close to the $K$ point. The dotted lines indicate all possible initial and final electronic states in the valence and conduction band for a specific momentum transfer $\left\{m_{x}, m_{y}\right\}=$ $\{2 \pi /(10 \mathrm{~nm}), 0\}$. (b) Sketch of the equienergy lines in the valence band of graphene around the $K$ point. The red line indicates all initial electronic states $\mathbf{k}_{\mathrm{v}}(\varphi)$ for a momentum transfer along $k_{x}$. (c) $\mathcal{M}_{\mathrm{el}-\mathrm{pl}}^{\mathbf{k}_{\mathrm{v}}, \mathbf{k}_{\mathrm{c}}}(\varphi)$ plotted around the $K$ point for the specific momentum transfer $m_{x}=1, m_{y}=0$ using the tight-binding model (TB) or the linear-band approximation (LB). (d) $\mathcal{M}_{\text {el-pt }}^{\mathbf{k}}(\varphi)$ plotted along an equienergy contour around the $K$ point. Parameters: Lattice of oblate Au spheroids with $r_{x y}=20 \mathrm{~nm}, r_{z}=10 \mathrm{~nm}, \Delta R=60 \mathrm{~nm}, R_{z}=$ $10 \mathrm{~nm} ; \boldsymbol{\varepsilon}_{\mathrm{pt}}=\mathbf{e}_{x}, \hbar \omega=2.3 \mathrm{eV}$, incident light power $1 \mathrm{~mW} / \mu \mathrm{m}^{2}$; tight-binding band structure from Ref. [8].

\section{LOCAL OPTICAL ABSORPTION IN PHASE SPACE}

For photon energies below $3 \mathrm{eV}$, optical excitations occur close the $K$ points in the Brillouin zone of graphene [13,42]. For a specific momentum transfer $\mathbf{k}_{\mathrm{c}}-\mathbf{k}_{\mathrm{v}}$ and energy of the light field $\hbar \omega$, all optical transitions that satisfy energy conservation $\epsilon_{\mathrm{el}}^{\mathrm{c}}\left(\mathbf{k}_{\mathrm{c}}\right)-\epsilon_{\mathrm{el}}^{\mathrm{v}}\left(\mathbf{k}_{\mathrm{v}}\right)=\hbar \omega$ form a ring around the $K$ point [Fig. 4(a)]. $\epsilon_{\mathrm{el}}^{\mathrm{v}}$ and $\epsilon_{\mathrm{el}}^{\mathrm{c}}$ are the energy dispersions of valence and conduction band. In Fig. 4(b) we plot the equienergy lines of the valence band as gray lines. For $\mathbf{k}_{\mathrm{c}}-\mathbf{k}_{\mathrm{v}} \neq 0$, the initial electronic states $\mathbf{k}_{\mathrm{v}}$ and final electronic states $\mathbf{k}_{\mathrm{c}}$ of the optical transitions are shifted with respect to the equienergy lines in the band structure [red line in Fig. 4(b)]. We define an angle $\varphi$ of the initial electronic state wave vector $\mathbf{k}_{\mathrm{v}}$ to the $k_{x}$ axis, 

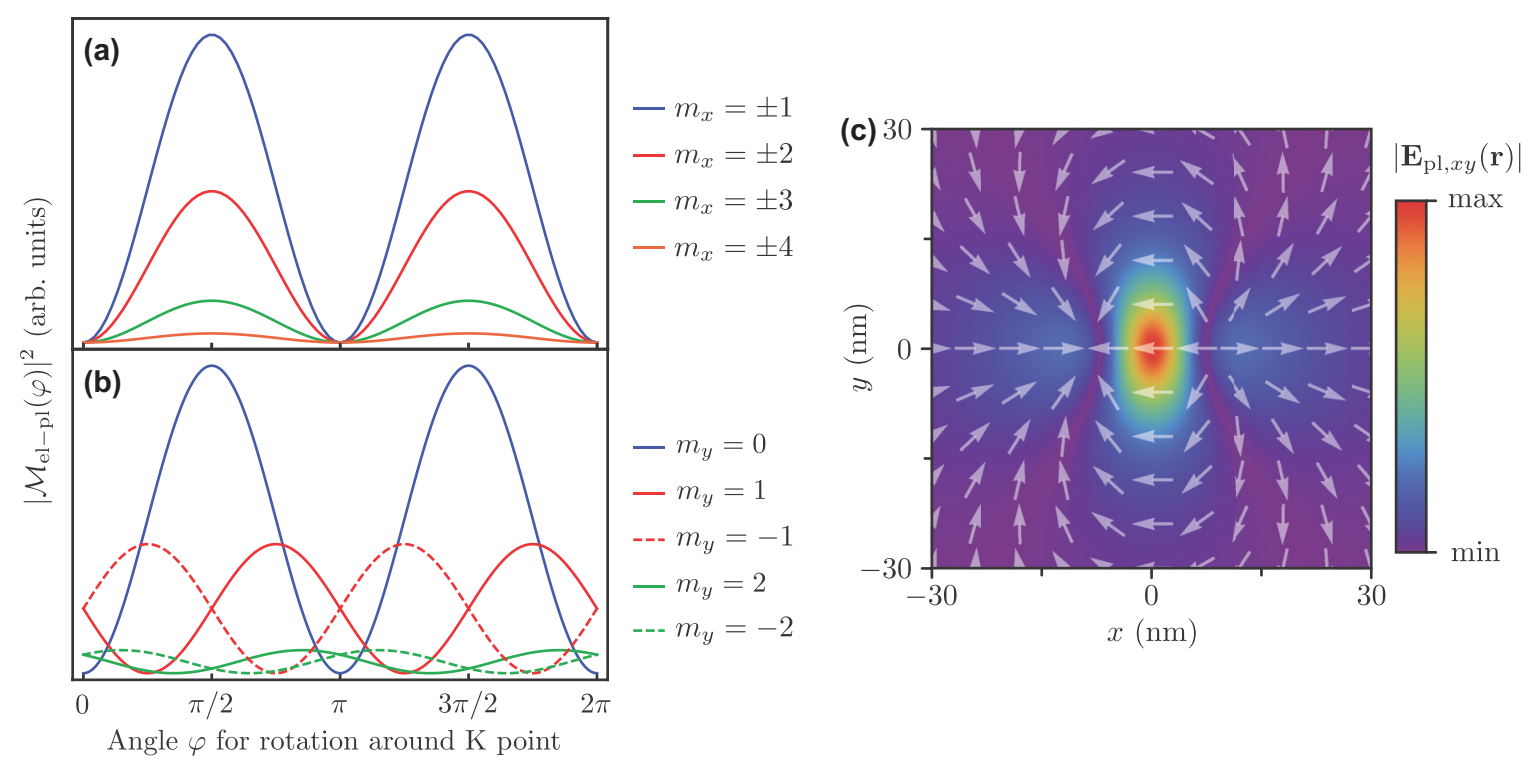

FIG. 5. Probability $\left|\mathcal{M}_{\mathrm{el}-\mathrm{pl}}^{\mathbf{k}_{\mathrm{v}}, \mathbf{k}_{\mathrm{c}}}(\varphi)\right|^{2}$ for optical transitions around the $K$ point for specific momentum transfers with (a) different $m_{x}$ and $m_{y}=0$ and (b) different $m_{y}$ and $m_{x}=1 . \varphi$ is the angle between the initial state wave vector $\mathbf{k}_{\mathrm{v}}$ and the $k_{x}$ axis, see Fig. 4(b). (c) Polarization of the plasmonic near field $\mathbf{E}_{\mathrm{pl}}(\mathbf{r})$ in the graphene plane. The vectors show the polarization direction and the contour plot the strength of the electric field. Parameters: Lattice of oblate Au spheroids with $r_{x y}=20 \mathrm{~nm}, r_{z}=10 \mathrm{~nm}, \Delta R=60 \mathrm{~nm}, R_{z}=10 \mathrm{~nm} ; \boldsymbol{\varepsilon}_{\mathrm{pt}}=\mathbf{e}_{x}, \hbar \omega=2.3 \mathrm{eV}$.

such that increasing $\varphi$ corresponds to a rotation around the $K$ point. In Fig. 4(c) we plot the plasmon-electron matrix element $\mathcal{M}_{\text {el-pl }}(\varphi)$ for a specific momentum transfer along $k_{x}$; the effect of other momentum transfers will be discussed later. For comparison, we also plot the photon-electron matrix element $\mathcal{M}_{\text {el-pt }}(\varphi)$ [Fig. 4(d)]. Overall, $\mathcal{M}_{\text {el-pl }}$ possesses the same functional dependence as $\mathcal{M}_{\text {el-pt }}$. While $\mathcal{M}_{\text {el-pt }}$ is a real number, $\mathcal{M}_{\text {el-pl }}$ is complex because of the complex optical polarizability $\alpha(\omega)$ of the metallic nanostructure. The real parts have opposite sign because most of the plasmonic near field in the graphene plane is opposite to the incident light field [see Fig. 5(c)]. We use either the linear-band approximation (LB) or a tight-binding model (TB) for the electronic wave functions and energy dispersion of graphene. There are only slight differences between both approximations [compare solid and dashed lines in Figs. 4(c) and 4(d)]. The trigonal warping of the electronic band structure induces an additional asymmetry in the angular dependence of the matrix elements. The magnitude of the matrix elements is highly inhomogeneous around the $K$ point and vanishes for two angles $\varphi$. It was previously shown for photon-electron interaction that these angles depend on the polarization of the light field $[9,13]$. For linearly polarized light, $\mathcal{M}_{\text {el-pt }}$ vanishes for momentum directions along the polarization of the incident light field and is maximum perpendicular to it.

The optical absorption probability is given by the square of the interaction matrix element. In Fig. 5 we illustrate the effect of different momentum transfers $\mathbf{k}_{\mathrm{c}}-\mathbf{k}_{\mathrm{v}}$ on the local absorption probability $\left|\mathcal{M}_{\mathrm{el}-\mathrm{pl}}(\varphi)\right|^{2}$ around the $K$ point. We consider a light polarization along $x$ and use the linear-band approximation. For a momentum transfer along $k_{x}$ the absorption probability is proportional to $\sin ^{2} \varphi$ and largest for $m_{x}= \pm 1$ and $m_{y}=0$ [Fig. 5(a)]. The combination $\left\{m_{x}, m_{y}\right\}$ for which the matrix element is largest depends on the light polarization, the spacing $\Delta R$ of the plasmonic dipoles, and their distance $R_{z}$ to the graphene lattice. In Fig. 5(b) we plot the transition probability for different momentum transfers along $k_{y}$. A momentum transfer solely along $k_{y}$ is forbidden for light polarization along $x$, because $\mathbf{p} \cdot\left(\mathbf{k}_{\mathrm{c}}-\mathbf{k}_{\mathrm{v}}\right)=0$ in Eq. (16). We therefore consider $m_{x}=1$. Interestingly, the angles $\varphi$ for which the transition probability vanishes are shifted by a momentum transfer along $k_{y}$, such that $\left|\mathcal{M}_{\mathrm{el}-\mathrm{pl}}(\varphi)\right|^{2} \propto \sin ^{2}(\varphi+\Delta \varphi)[$ Fig. 5(b)].

The shift $\Delta \varphi$ of the nodes in the optical absorption can be understood by the polarization of the local electric field $\mathbf{E}_{\mathrm{pl}, x y}(\mathbf{r})$ that is generated by the plasmonic nanostructure in the graphene plane [see Fig. 5(c)]. For a polarization along $x$, the nodes occur at $k_{x}=0$, i.e., at the angles $\varphi=0$ and $\pi$, and for a polarization along $y$ at $k_{y}=0$ (i.e., $\varphi=\pi / 2$ and $3 \pi / 2$ ) [13]. The shift $\Delta \varphi$ in Fig. 5(b) is therefore attributed to the components of the plasmonic near field that are polarized along $y$. This can be made more clear by writing the electric field $\mathbf{E}_{\mathrm{pl}, x y}(\mathbf{r})$ in terms of its Fourier components. Using Eq. (14) and $\mathbf{E}_{\mathrm{pl}}(\mathbf{r})=-\nabla \varphi_{\mathrm{pl}}(\mathbf{r})$ we obtain

$$
\mathbf{E}_{\mathrm{pl}, x y}(\mathbf{r})=\frac{\mathcal{N}_{\mathrm{pl}}}{2 \epsilon_{0}} \sum_{\Delta \mathbf{q} \neq 0} \frac{\mathbf{p} \cdot \Delta \mathbf{q}}{|\Delta \mathbf{q}|} e^{i \Delta q_{x} x} e^{i \Delta q_{y} y} e^{-|\Delta \mathbf{q}|\left|R_{z}\right|} \Delta \mathbf{q} .
$$

The polarization of each Fourier component $\tilde{\mathbf{E}}_{\mathrm{pl}, x y}(\mathbf{r}, \Delta \mathbf{q})$ is parallel to the spatial frequency $\Delta \mathbf{q}$, which equals the momentum transfer $\mathbf{k}_{\mathrm{c}}-\mathbf{k}_{\mathrm{v}}$ of an optical transition. A momentum transfer along $k_{x}$ therefore corresponds to a Fourier component with polarization along $x$ and a momentum transfer along $k_{y}$ corresponds to a Fourier component with polarization along $y$.

In Fig. 6 we plot the total angular transition probability

$$
W_{\mathrm{el}-\mathrm{pl}}(\varphi)=\sum_{m_{x}, m_{y}}\left|\mathcal{M}_{\mathrm{el}-\mathrm{pl}}^{\mathbf{k}_{\mathrm{v}} \mathbf{k}_{\mathrm{c}}}\left(m_{x}, m_{y}, \varphi\right)\right|^{2},
$$

which is the probability of all transitions associated with the angle $\varphi$ of the initial state wave vector $\mathbf{k}_{\mathrm{v}}$ to the $k_{x}$ axis. The transition amplitudes are summed incoherently, i.e., the matrix 


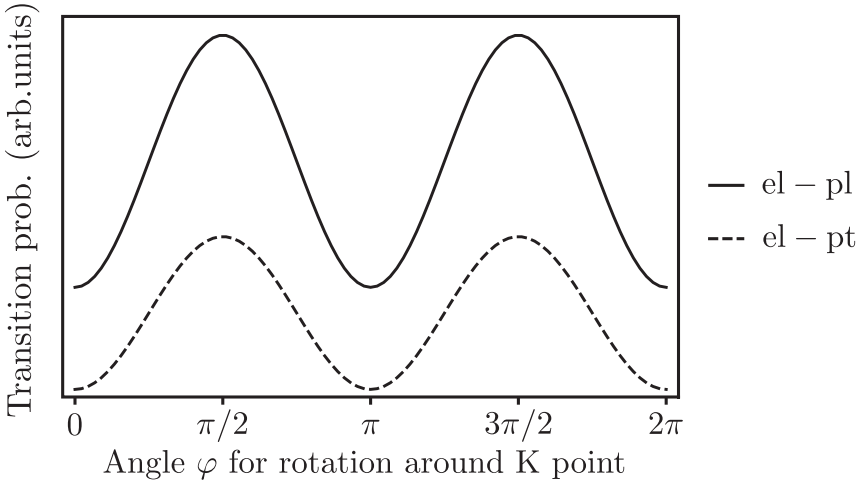

FIG. 6. Probability of all optical transitions associated with an angle $\varphi$ of the initial state wave vector $\mathbf{k}_{\mathrm{v}}$ to the $k_{x}$ axis in the presence of plasmonic enhancement (solid line) and without plasmonic enhancement (dashed line). Parameters: Lattice of oblate Au spheroids with $r_{x y}=20 \mathrm{~nm}, r_{z}=10 \mathrm{~nm}, \Delta R=60 \mathrm{~nm}, R_{z}=10 \mathrm{~nm} ; \boldsymbol{\varepsilon}_{\mathrm{pt}}=\mathbf{e}_{x}$, $\hbar \omega=2.3 \mathrm{eV}$.

element is squared before calculating the sum, because each optical transition has a different final state. For comparison, we also plot the transition probability $W_{\text {el-pt }}(\varphi)=\left|\mathcal{M}_{\text {el-pt }}(\varphi)\right|^{2}$ for direct light absorption. While $W_{\mathrm{el}-\mathrm{pt}}$ vanishes for $\varphi=0$ and $\pi$, this is no longer the case in the presence of plasmonic enhancement (compare solid and dashed lines in Fig. 6). $W_{\text {el-pl }}$ is finite for all angles $\varphi$ because the plasmonic near field $\mathbf{E}_{\mathrm{pl}, x y}(\mathbf{r})$ is not solely polarized along $x$. It contains the incoherent sum over transition amplitudes that are associated with different optical polarizations of $\mathbf{E}_{\mathrm{pl}, x y}(\mathbf{r})$ [i.e., the sum of the curves in Figs. 5(a) and 5(b)]. This clearly shows that in phase space the plasmon-mediated optical absorption is not just the product of an enhancement factor with the direct optical absorption.

\section{INTEGRATED OPTICAL ABSORPTION}

We now use the expression for $\mathcal{M}_{\text {el-pl }}$ in Eq. (16) to calculate the integrated plasmon-enhanced optical absorption of graphene $P_{\mathrm{abs}}^{\mathrm{el}-\mathrm{pl}}(\omega)$, which is, e.g., relevant for the photocurrent enhancement in graphene-based photonic devices [26-28]. Using perturbation theory, we obtain [38]

$$
P_{\mathrm{abs}}^{\mathrm{el}-\mathrm{pl}}(\omega)=\frac{2 \hbar \omega \mu_{0} c}{E_{0}^{2}} g_{\mathrm{s}} \iint \frac{d k_{\mathrm{v}, x} d k_{\mathrm{v}, y}}{(2 \pi)^{2}} \sum_{\mathbf{k}_{\mathrm{c}}} \eta_{\mathbf{k}_{\mathrm{v}}, \mathbf{k}_{\mathrm{c}}}^{\mathrm{el}-\mathrm{pl}},
$$

with the transition rate

$$
\eta_{\mathbf{k}_{\mathrm{v}}, \mathbf{k}_{\mathrm{c}}}^{\mathrm{el}}=\frac{2 \pi}{\hbar}\left|\mathcal{M}_{\mathrm{el}-\mathrm{pl}}^{\mathbf{k}_{\mathrm{v}}, \mathbf{k}_{\mathrm{c}}}\right|^{2} \delta\left[\epsilon_{\mathrm{el}}^{\mathrm{c}}\left(\mathbf{k}_{\mathrm{c}}\right)-\epsilon_{\mathrm{el}}^{\mathrm{v}}\left(\mathbf{k}_{\mathrm{v}}\right)-\hbar \omega\right] .
$$

The integration and summation is carried out over the entire Brillouin zone of graphene or alternatively for low-energy excitations around one $K$ point. For the latter case one has to multiply the expression in Eq. (22) by the valley degeneracy $g_{\mathrm{v}}=2$. The integral and sum over all $\mathbf{k}_{\mathrm{v}}$ and $\mathbf{k}_{\mathrm{c}}$ is calculated after squaring the matrix element $\mathcal{M}_{\text {el-pl }}$ because each optical transition has a different final state. The factor $g_{\mathrm{s}}=2$ accounts for spin degeneracy and the Delta function for energy conservation.

Equation (22) accounts only for the plasmon-mediated optical transitions and neglects transitions that are induced by direct absorption of the incident light field. The enhanced optical absorption of graphene is calculated by summing the plasmon-mediated absorption $P_{\mathrm{abs}}^{\mathrm{el}-\mathrm{pl}}(\omega)$ and the direct optical absorption $P_{\mathrm{abs}}^{\mathrm{el}-\mathrm{pt}}(\omega)$ incoherently, because of the different initial and final states. More precisely, $P_{\mathrm{abs}}^{\mathrm{el}-\mathrm{pt}}$ involves only vertical optical transitions because the photon momentum is perpendicular to the graphene sheet, whereas $P_{\mathrm{abs}}^{\mathrm{el}-\mathrm{pl}}$ involves only nonvertical transitions due to the momentum transfer $\Delta \mathbf{q}$ by the plasmonic lattice with the selection rule $\Delta \mathbf{q} \neq 0$.

The enhancement of the optical absorption is calculated as $\operatorname{Enh}(\omega)=\left[P_{\mathrm{abs}}^{\mathrm{el}-\mathrm{pl}}(\omega)+P_{\mathrm{abs}}^{\mathrm{el}-\mathrm{pt}}(\omega)\right] / P_{\mathrm{abs}}^{\mathrm{el}-\mathrm{pt}}(\omega)$. Using Eqs. (16), (22), and (23) and the linear-band approximation, we obtain the explicit expression [38]

$$
\operatorname{Enh}(\omega)=1+\frac{4 \pi^{2}|\alpha(\omega)|^{2}}{\Delta R^{4}} \sum_{\Delta \mathbf{q}}\left|\boldsymbol{\varepsilon}_{\mathrm{pt}} \cdot \Delta \mathbf{q}\right|^{2} e^{-2|\Delta \mathbf{q}|\left|R_{z}\right|} .
$$

The enhancement only depends on parameters that are specific for the plasmonic nanostructure and excitation of the LSPR. Except for the distance $R_{z}$ of graphene to the plasmonic lattice, any graphene-specific parameters canceled.

The excitation-energy dependence of the plasmonenhanced optical absorption is plotted in Fig. 7(a). While the intrinsic optical absorption is approximately constant (blue lines), the plasmonic lattice induces a resonance which is given by the polarizability $|\alpha(\omega)|^{2}$ of the metallic nanostructure (red lines). The energy of the resonance depends on the material, shape, and coupling of the metallic nanoparticles. There is overall a good agreement between the full tight-binding calculations (solid lines) and the linear-band approximation (dashed lines). The tight-binding calculations deviate from the linear-band approximation with increasing excitation energy because of the trigonal warping and nonlinearity of the electronic band structure [5]. The deviations are similar for the plasmon-enhanced and the intrinsic optical absorption and vanish when calculating the enhancement [Fig. 7(b)]. This shows that the plasmonic enhancement is independent from graphene-specific material parameters and Eq. (24) is valid independent of the linear-band approximation.

In our microscopic model of optical absorption enhancement, the scattering amplitudes that are either associated with plasmon-mediated transitions or direct transitions were summed incoherently because of different final states. This appears to contradict the macroscopic (electromagnetic) approach, where the optical absorption is considered to be proportional to the coherent sum of the incident electric field $\mathbf{E}_{\mathrm{inc}}=E_{0} \varepsilon_{\mathrm{pt}}$ and the plasmonic near field $\mathbf{E}_{\mathrm{pl}}(\mathbf{r})$, i.e., $\left|\mathbf{E}_{\text {inc }}+\mathbf{E}_{\mathrm{pl}}(\mathbf{r})\right|^{2}$ [25-28]. In the following we show that both models are consistent and Eq. (24) can be also derived within a purely electromagnetic theory.

The integrated enhancement of the optical absorption is calculated within the macroscopic theory as

$$
\operatorname{Enh}^{\mathrm{EM}}(\omega)=\iint_{-\Delta R / 2}^{\Delta R / 2} d x d y \frac{\left|\mathbf{E}_{\mathrm{inc}}+\mathbf{E}_{\mathrm{pl}, x y}(\mathbf{r}, \omega)\right|^{2}}{\Delta R^{2} E_{0}^{2}}
$$

The $z$ component of the plasmonic near field is dropped because graphene can only interact with the in-plane components $\mathbf{E}_{\mathrm{pl}, x y}$. Next, we rewrite the plasmonic near field in terms of its Fourier components $\mathbf{E}_{\mathrm{pl}, x y}(\mathbf{r}, \omega)=\sum_{\Delta \mathbf{q}} \tilde{\mathbf{E}}_{\mathrm{pl}, x y}(\mathbf{r}, \Delta \mathbf{q})$. 


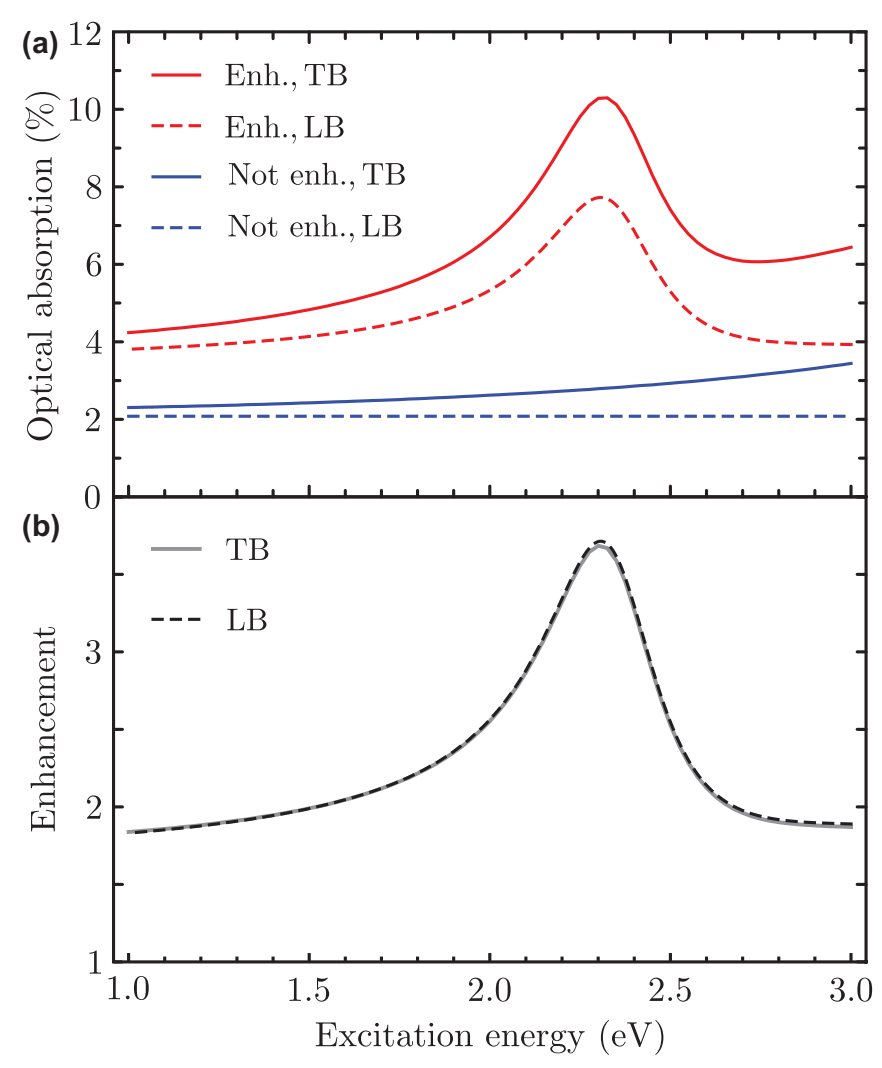

FIG. 7. (a) Optical absorption of graphene as a function of excitation energy with plasmonic enhancement (red) and without plasmonic enhancement (blue), calculated either with the linear-band approximation (dashed, LB) or with a tight-binding model (solid, TB). (b) Enhancement of the optical absorption calculated with a tightbinding model (solid) or the linear-band approximation (dashed). Parameters: Lattice of oblate Au spheroids with $r_{x y}=20 \mathrm{~nm}, r_{z}=$ $10 \mathrm{~nm}, \Delta R=60 \mathrm{~nm}, R_{z}=10 \mathrm{~nm}, \boldsymbol{\varepsilon}_{\mathrm{pt}}=\mathbf{e}_{x}$; tight-binding band structure from Ref. [8].

While the incident electric field $\mathbf{E}_{\text {inc }}$ is constant, each Fourier component of the plasmonic near field $\tilde{\mathbf{E}}_{\mathrm{pl}, x y}$ oscillates with a spatial frequency $\Delta \mathbf{q}=2 \pi / \Delta R\left\{m_{x}, m_{y}\right\}$ that satisfies periodic boundary conditions at the border of the integration area, see Eq. (20). Consequently, the interference term of $\left|\mathbf{E}_{\text {inc }}+\mathbf{E}_{\mathrm{pl}, x y}(\mathbf{r}, \omega)\right|^{2}$ vanishes when calculating the integral in Eq. (25). We obtain

$$
\begin{aligned}
& \iint_{-\Delta R / 2}^{\Delta R / 2} d x d y\left|\mathbf{E}_{\mathrm{inc}}+\mathbf{E}_{\mathrm{pl}, x y}(\mathbf{r}, \omega)\right|^{2} \\
& \quad=\iint_{-\Delta R / 2}^{\Delta R / 2} d x d y\left|\mathbf{E}_{\mathrm{inc}}\right|^{2}+\left|\mathbf{E}_{\mathrm{pl}, x y}(\mathbf{r}, \omega)\right|^{2} .
\end{aligned}
$$

The coherent and the incoherent sum of the field amplitudes $\mathbf{E}_{\text {inc }}$ and $\mathbf{E}_{\mathrm{pl}, x y}$ are identical when calculating the integrated optical absorption enhancement. The same argument can be made about the Fourier components $\tilde{\mathbf{E}}_{\mathrm{pl}, x y}(\mathbf{r}, \Delta \mathbf{q})$ of the plasmonic near field. As a result of the microscopic approach, each Fourier component corresponds to optical transitions with a different momentum transfer $\mathbf{k}_{\mathrm{c}}-\mathbf{k}_{\mathrm{v}}=\Delta \mathbf{q}$. The matrix elements $\mathcal{M}_{\text {el-pl }}^{\mathbf{k}_{\mathrm{v}}, \mathbf{k}_{\mathrm{c}}}$ were summed incoherently in Eq. (22). Indeed all interference terms of $\left|\sum_{\Delta \mathbf{q}} \tilde{\mathbf{E}}_{\mathrm{pl}, x y}(\mathbf{r}, \Delta \mathbf{q})\right|^{2}$ vanish when calculating the integral in Eq. (25), such that

$$
\begin{aligned}
& \iint_{-\Delta R / 2}^{\Delta R / 2} d x d y\left|\sum_{\Delta \mathbf{q}} \tilde{\mathbf{E}}_{\mathrm{pl}, x y}(\mathbf{r}, \Delta \mathbf{q})\right|^{2} \\
& =\iint_{-\Delta R / 2}^{\Delta R / 2} d x d y \sum_{\Delta \mathbf{q}}\left|\tilde{\mathbf{E}}_{\mathrm{pl}, x y}(\mathbf{r}, \Delta \mathbf{q})\right|^{2} .
\end{aligned}
$$

Since $\tilde{\mathbf{E}}_{\mathrm{pl}, x y}(\mathbf{r}, \Delta \mathbf{q}) \propto e^{i \Delta q_{x} x} e^{i \Delta q_{y} y}$, the position dependence of each component becomes zero when calculating the absolute square and we find

$$
\operatorname{Enh}^{\mathrm{EM}}(\omega)=1+\sum_{\Delta \mathbf{q}}\left|\tilde{\mathbf{E}}_{\mathrm{pl}, x y}(\mathbf{r}, \Delta \mathbf{q})\right|^{2} / E_{0}^{2},
$$

which is identical with the enhancement obtained within the microscopic approach, see Eq. (24). We thereby confirm that the integrated plasmon-enhanced optical absorption in graphene is the product of the intrinsic absorption with the purely electromagnetic enhancement factor in Eq. (25). The electromagnetic enhancement factor can be also calculated for more complex plasmonic nanostructures using standard numerical techniques, such as the finite-difference time domain or the finite-elements method [43].

\section{EFFECTS OF FIELD RETARDATION}

All analytic expressions that were derived so far were based on the quasistatic approximation because we aimed to obtain compact and comprehensible expressions. The sizes of the nanoparticles in previous experimental works were typically on the order of $100 \mathrm{~nm}$ or even larger so that field retardation can in general not be neglected [25-28]. The aim of this section is to estimate how a generalization of the equations beyond the quasistatic approximation affects the main conclusions of the paper.

In Eq. (1) we approximated the electric field of the plasmonic nanoparticle lattice with the electrostatic potential $\phi_{\mathrm{pl}}$, which can be derived from the Poisson equation $\nabla^{2} \phi_{\mathrm{pl}}(\mathbf{r})=-\rho(\mathbf{r}) / \epsilon_{0}$. By using the Lorentz gauge $\phi_{\mathrm{pl}}(\mathbf{r})=-i \omega / k_{\mathrm{pt}}^{2} \nabla_{\mathbf{r}} \cdot \mathbf{A}_{\mathrm{pl}}(\mathbf{r})$ and the Helmholtz equation $\left(\nabla^{2}+k_{\mathrm{pt}}^{2}\right) \phi_{\mathrm{pl}}(\mathbf{r})=-\rho(\mathbf{r}) / \epsilon_{0}$ instead of the Poisson equation we account for the finite wavelength of the light (given by the wave number $\left.k_{\mathrm{pt}}=2 \pi / \lambda\right)$ and obtain [38]

$$
\phi_{\mathrm{pl}}^{\text {ret }}(\mathbf{r})=\frac{\mathcal{N}_{\mathrm{pl}}}{2 \epsilon_{0}} \sum_{\Delta \mathbf{q}} \frac{\mathbf{p} \cdot \Delta \mathbf{q}}{\sqrt{k_{\mathrm{pt}}^{2}-|\Delta \mathbf{q}|^{2}}} e^{i \Delta \mathbf{q} \cdot\{x, y\}} e^{i \sqrt{k_{\mathrm{pt}}^{2}-|\Delta \mathbf{q}|^{2}}\left|R_{z}\right|} .
$$

This expression reduces to Eq. (14) for the approximation $k_{\mathrm{pt}}=$ 0 . Furthermore, we also have to account for the vector potential (for a derivation see the Supplemental Material [38])

$$
\mathbf{A}_{\mathrm{pl}}^{\mathrm{ret}}(\mathbf{r})=\frac{k_{\mathrm{pt}}^{2} \mathcal{N}_{\mathrm{pl}} \mathbf{p}}{2 \omega \epsilon_{0}} \sum_{\Delta \mathbf{q}} e^{i \Delta \mathbf{q} \cdot\{x, y\}} \frac{e^{i \sqrt{k_{\mathrm{pt}}^{2}-|\Delta \mathbf{q}|^{2}}\left|R_{z}\right|}}{\sqrt{k_{\mathrm{pt}}^{2}-|\Delta \mathbf{q}|^{2}}} .
$$

In contrast to the scalar potential, the vector potential has a nonvanishing Fourier component with $\Delta \mathbf{q}=0$ that corresponds to the radiation of a plane electromagnetic wave into the far field. 


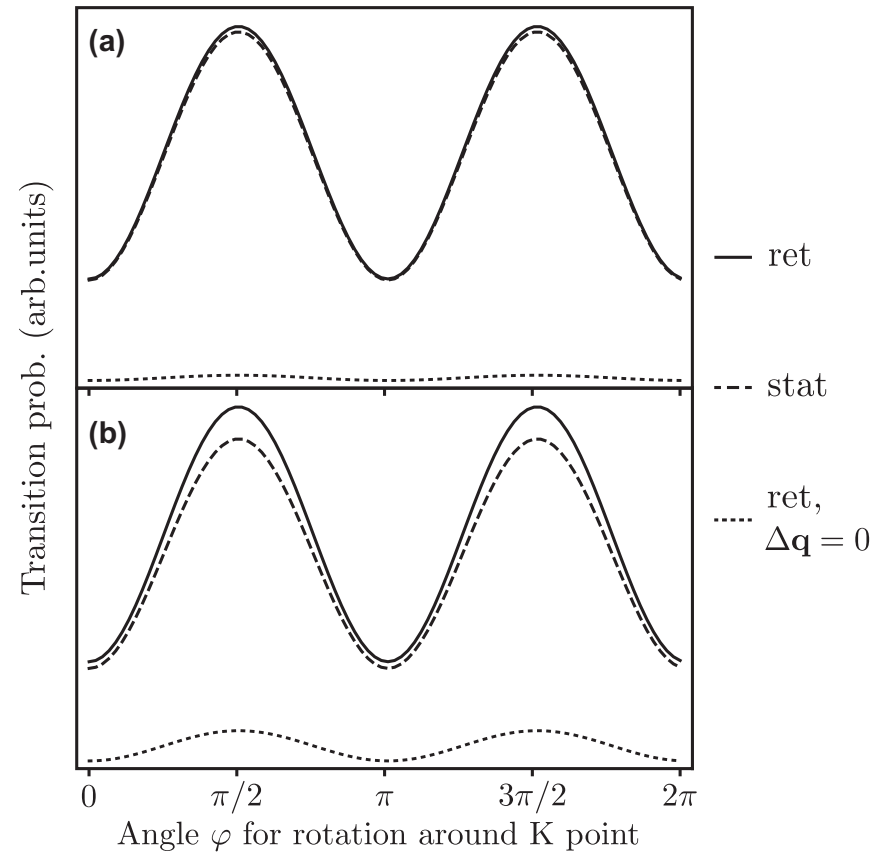

FIG. 8. Effect of field retardation on the transition probability around the $K$ point. Solid lines show the probability of all plasmonmediated transitions for retarded fields (ret) and dashed lines for the quasistatic approximation (stat). The dotted lines show the probability for plasmon-mediated vertical transitions with $\Delta \mathbf{q}=0$ that are allowed for retarded fields. (a) Lattice of oblate Au spheroids with $r_{x y}=20 \mathrm{~nm}, r_{z}=10 \mathrm{~nm}, \Delta R=60 \mathrm{~nm}, R_{z}=10 \mathrm{~nm} ; \boldsymbol{\varepsilon}_{\mathrm{pt}}=\mathbf{e}_{x}$, $\hbar \omega=2.3 \mathrm{eV}$. (b) All size parameters of (a) are scaled by a factor of 2.5 .

We calculate the plasmon-electron matrix element with the interaction Hamiltonian

$$
\mathcal{H}_{\mathrm{el}-\mathrm{pl}}^{\mathrm{ret}}(\mathbf{r})=-\frac{e}{2} \phi_{\mathrm{pl}}(\mathbf{r})-\frac{i \hbar e}{2 m} \mathbf{A}_{\mathrm{pl}}(\mathbf{r}) \cdot \nabla_{\mathbf{r}} .
$$

This Hamiltonian is obtained from Eq. (9) by neglecting the $\nabla_{\mathbf{r}} \cdot \mathbf{A}_{\mathrm{pl}}(\mathbf{r})$ term as it is by a factor $\hbar \omega / 2 m c^{2} \approx 10^{-6}$ smaller than the $\phi_{\mathrm{pl}}$ term when using the Lorentz gauge. The analytic expression and derivation of the matrix element can be found in the Supplemental Material [38]. We obtain the same selection rules for the plasmon-mediated optical transitions as before, with the exception that vertical optical transitions can be excited by the far field that is emitted by the plasmonic lattice.

In Fig. 8 we plot the probability of the plasmon-mediated optical transitions around the $K$ point, similar to Fig. 6 above; see also Eq. (21). The solid lines show the transition probability when accounting for field retardation and the dashed lines for the quasistatic approximation. We also plot the probability for plasmon-mediated vertical transitions with $\Delta \mathbf{q}=0$ which are only allowed for the retarded case. For a particle diameter of $40 \mathrm{~nm}$ and a lattice constant of $60 \mathrm{~nm}$, which we considered above, the effect of retardation is negligible [Fig. 8(a)]. However, when scaling all size parameters by a factor of 2.5, which resembles better the case of previous experiments [25-27], the transition probability is larger for the retarded case than for the quasistatic approximation [Fig. 8(b)]. The probability of plasmon-mediated vertical transitions is no longer negligible. Despite these quantitative differences, the

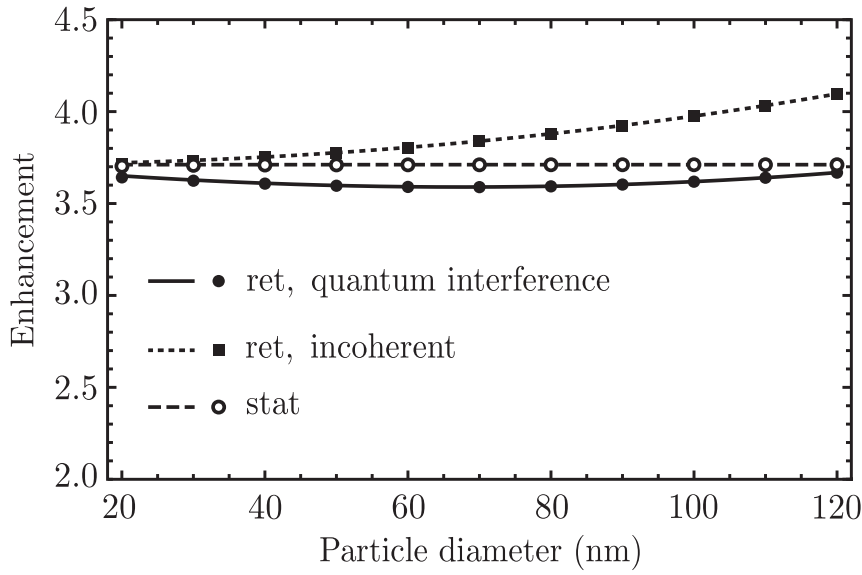

FIG. 9. Maximum optical absorption enhancement as function of the particle diameter, accounting for retardation and quantum interference (solid line, disks), for retardation but not for quantum interference (dotted line, squares) and within the quasistatic approximation (dashed line, circles). The enhancement is calculated, both, with the microscopic theory (symbols) and with the macroscopic theory (lines). All size parameters, i.e., $r_{z}, \Delta R$, and $R_{z}$, were scaled by the same factor as the particle diameter $2 r_{x y}$. Parameters for $20 \mathrm{~nm}$ particle diameter: Lattice of oblate Au spheroids with $r_{x y}=10 \mathrm{~nm}$, $r_{z}=5 \mathrm{~nm}, \Delta R=30 \mathrm{~nm}, R_{z}=5 \mathrm{~nm} ; \boldsymbol{\varepsilon}_{\mathrm{pt}}=\mathbf{e}_{x}, \hbar \omega=2.3 \mathrm{eV}$.

transition probability remains finite for all angles $\varphi$ and the conclusions drawn in Sec. IV remain valid.

When accounting for the far field of the plasmonic nanostructure, the enhancement of the optical absorption can no longer be calculated by summing the plasmon-mediated and the direct optical absorption incoherently, i.e., $\operatorname{Enh}(\omega)=$ $\left[P_{\mathrm{abs}}^{\mathrm{el}-\mathrm{pl}}(\omega)+P_{\mathrm{abs}}^{\mathrm{el}-\mathrm{pt}}(\omega)\right] / P_{\mathrm{abs}}^{\mathrm{el}-\mathrm{pt}}(\omega)$. For each direct optical transition with wave vector $\mathbf{k}$ that is induced by the incident light field there exists a plasmon-induced transition with $\Delta \mathbf{q}=0$ that has the same initial and final electronic state. This leads to quantum interference between both absorption pathways, i.e., the transition probability is given by $\left|\mathcal{M}_{\mathrm{el}-\mathrm{pt}}^{\mathbf{k}}+\mathcal{M}_{\mathrm{el}-\mathrm{pl}}^{\mathbf{k}}\right|^{2}$ and not by $\left|\mathcal{M}_{\text {el-pt }}^{\mathbf{k}}\right|^{2}+\left|\mathcal{M}_{\text {el-pl }}^{\mathbf{k} \text {,ff }}\right|^{2}$, where $\mathcal{M}_{\text {el-pl }}^{\mathbf{k}, \text { ff }}$ is the component of the plasmon-electron matrix element with $\Delta \mathbf{q}=0$.

To estimate the effect of field retardation and quantum interference, we plot the maximum optical absorption enhancement as a function of the size of the plasmonic nanostructure in Fig. 9 (see the Supplemental Material for details on the calculation [38]). We obtain perfect agreement between the enhancement calculated with the microscopic and the macroscopic approach; compare symbols and lines in Fig. 9. The major conclusion drawn in Sec. V, that the plasmon-enhanced optical absorption of graphene is the intrinsic optical absorption multiplied by a purely electromagnetic enhancement factor, therefore remains also valid for retarded fields.

Interestingly, the enhancement factor that includes field retardation (solid line) deviates less than 3\% from the quasistatic approximation (dashed line). From Fig. 8(b) one would expect an increase in enhancement when accounting for field retardation. The quasistatic approximation, however, slightly overestimates the enhancement factor (Fig. 9). This is explained by destructive quantum interference between different absorption pathways as can be seen from a comparison to 
an enhancement factor that neglects quantum interference (dotted line). The far field that is emitted by the plasmonic nanostructure $\mathbf{E}_{\mathrm{pl}}^{\mathrm{ret}, \mathrm{ff}}=2 \pi i k_{\mathrm{pt}} \mathcal{N}_{\mathrm{pl}} \alpha_{\mathrm{pl}}(\omega) E_{0} \boldsymbol{\varepsilon}_{\mathrm{pt}}$ partly cancels the incoming light field $\mathbf{E}_{\mathrm{inc}}=E_{0} \boldsymbol{\varepsilon}_{\mathrm{pt}}$ in the graphene plane, which leads to a decrease of the field enhancement. We find that the quasistatic approximation can be used to estimate the optical absorption enhancement independent of the size of the plasmonic nanoparticles and their spacing, for the geometry that is considered in this paper. Overall, the main conclusions that were drawn in the previous sections remain valid for a generalization beyond the quasistatic approximation.

\section{CONCLUSIONS}

We presented a microscopic theory for the interaction of graphene with a lattice of metallic nanoparticles that supports localized surface plasmon resonances. The electric near field of the plasmonic excitation induces indirect optical interband transitions in graphene with a momentum transfer that depends on the localization of the plasmonic near field and the periodicity of the metallic nanostructure. The population of photoexcited carriers in phase space depends on the polarization pattern of the plasmonic near field in the graphene plane and differs from the one generated by the incident light field. We also drawed a connection to a purely electromagnetic theory of the optical absorption enhancement. The momentum transfer of each plasmon-mediated optical transition corresponds to the spatial frequency of a Fourier component of the plasmonic near field in the graphene plane. We obtain the same expression for the integrated optical absorption enhancement using the microscopic and the macroscopic approach. Our work leads to a better understanding of the photocarrier generation in graphene that is coupled to plasmonic nanostructures. The analytic expression for the plasmon-electron matrix element can be used in future works to calculate the plasmon-enhanced Raman spectrum of graphene and to study the carrier dynamics in hybrid graphene-metallic nanostructure optoelectronic devices.

\section{ACKNOWLEDGMENTS}

The authors thankfully acknowledge helpful discussions with Kirill Bolotin and Bruno Gondim de Melo Vieira. N.S.M. acknowledges Deutsche Telekom Stiftung for financial support. Parts of this work were supported by the European Research Council under grant DarkSERS (772108).
[1] R. Binder, Optical Properties of Graphene (World Scientific, Singapore, 2017).

[2] F. H. Koppens, M. B. Lundeberg, M. Polini, T. Low, and P. Avouris, in 2D Materials: Properties and Devices, edited by $\mathrm{P}$. Avouris, T. F. Heinz, and T. Low (Cambridge University Press, Cambridge, 2017), p. 104.

[3] F. Bonaccorso, Z. Sun, T. Hasan, and A. C. Ferrari, Nat. Photon. 4, 611 (2010).

[4] Z. Sun, A. Martinez, and F. Wang, Nat. Photon. 10, 227 (2016).

[5] R. R. Nair, P. Blake, A. N. Grigorenko, K. S. Novoselov, T. J. Booth, T. Stauber, N. M. R. Peres, and A. K. Geim, Science 320, 1308 (2008).

[6] K. F. Mak, M. Y. Sfeir, Y. Wu, C. H. Lui, J. A. Misewich, and T. F. Heinz, Phys. Rev. Lett. 101, 196405 (2008).

[7] T. Stroucken and S. W. Koch, Optical Properties of Graphene (World Scientific, Singapore, 2016), Chap. 2, pp. 43-84.

[8] S. Reich, J. Maultzsch, C. Thomsen, and P. Ordejón, Phys. Rev. B 66, 035412 (2002).

[9] E. Malic, T. Winzer, E. Bobkin, and A. Knorr, Phys. Rev. B 84, 205406 (2011).

[10] P. Venezuela, M. Lazzeri, and F. Mauri, Phys. Rev. B 84, 035433 (2011).

[11] C. Thomsen and S. Reich, Phys. Rev. Lett. 85, 5214 (2000).

[12] C.-F. Chen, C.-H. Park, B. W. Boudouris, J. Horng, B. Geng, C. Girit, A. Zettl, M. F. Crommie, R. A. Segalman, S. G. Louie, and F. Wang, Nature (London) 471, 617 (2011).

[13] A. Grüneis, R. Saito, G. G. Samsonidze, T. Kimura, M. A. Pimenta, A. Jorio, A. G. Souza Filho, G. Dresselhaus, and M. S. Dresselhaus, Phys. Rev. B 67, 165402 (2003).

[14] M. Mittendorff, T. Winzer, E. Malic, A. Knorr, C. Berger, W. A. de Heer, H. Schneider, M. Helm, and S. Winnerl, Nano Lett. 14, 1504 (2014).
[15] X.-Q. Yan, J. Yao, Z.-B. Liu, X. Zhao, X.-D. Chen, C. Gao, W. Xin, Y. Chen, and J.-G. Tian, Phys. Rev. B 90, 134308 (2014).

[16] M. Trushin, A. Grupp, G. Soavi, A. Budweg, D. De Fazio, U. Sassi, A. Lombardo, A. C. Ferrari, W. Belzig, A. Leitenstorfer, and D. Brida, Phys. Rev. B 92, 165429 (2015).

[17] J. C. König-Otto, M. Mittendorff, T. Winzer, F. Kadi, E. Malic, A. Knorr, C. Berger, W. A. de Heer, A. Pashkin, H. Schneider, M. Helm, and S. Winnerl, Phys. Rev. Lett. 117, 087401 (2016).

[18] T. Danz, A. Neff, J. H. Gaida, R. Bormann, C. Ropers, and S. Schäfer, Phys. Rev. B 95, 241412 (2017).

[19] S. Aeschlimann, R. Krause, M. Chávez-Cervantes, H. Bromberger, R. Jago, E. Malić, A. Al-Temimy, C. Coletti, A. Cavalleri, and I. Gierz, Phys. Rev. B 96, 020301 (2017).

[20] T. J. Echtermeyer, P. S. Nene, M. Trushin, R. V. Gorbachev, A. L. Eiden, S. Milana, Z. Sun, J. Schliemann, E. Lidorikis, K. S. Novoselov, and A. C. Ferrari, Nano Lett. 14, 3733 (2014).

[21] V. Georgakilas, M. Otyepka, A. B. Bourlinos, V. Chandra, N. Kim, K. C. Kemp, P. Hobza, R. Zboril, and K. S. Kim, Chem. Rev. 112, 6156 (2012).

[22] A. N. Grigorenko, M. Polini, and K. S. Novoselov, Nat. Photon. 6, 749 (2012).

[23] G. Berghäuser and E. Malić, Carbon 69, 536 (2014).

[24] M. Feierabend, G. Berghaeuser, A. Knorr, and E. Malic, Nat. Commun. 8, 14776 (2017).

[25] F. Schedin, E. Lidorikis, A. Lombardo, V. G. Kravets, A. K. Geim, A. N. Grigorenko, K. S. Novoselov, and A. C. Ferrari, ACS Nano 4, 5617 (2010).

[26] T. J. Echtermeyer, L. Britnell, P. K. Jasnos, A. Lombardo, R. V. Gorbachev, A. N. Grigorenko, A. K. Geim, A. C. Ferrari, and K. S. Novoselov, Nat. Commun. 2, 458 (2011).

[27] Y. Liu, R. Cheng, L. Liao, H. Zhou, J. Bai, G. Liu, L. Liu, Y. Huang, and X. Duan, Nat. Commun. 2, 579 (2011). 
[28] Z. Fang, Z. Liu, Y. Wang, P. M. Ajayan, P. Nordlander, and N. J. Halas, Nano Lett. 12, 3808 (2012).

[29] T. Hosseini and N. A. Kouklin, Appl. Phys. Lett. 105, 043104 (2014).

[30] R. Jago, F. Wendler, and E. Malic, Phys. Rev. B 96, 085431 (2017).

[31] P. G. Etchegoin, E. C. Le Ru, and M. Meyer, J. Chem. Phys. 125, 164705 (2006).

[32] P. B. Johnson and R. W. Christy, Phys. Rev. B 6, 4370 (1972).

[33] E. C. L. Ru and P. G. Etchegoin, Principles of Surface-Enhanced Raman Spectroscopy (Elsevier, Amsterdam, 2009).

[34] A. D. Humphrey and W. L. Barnes, Phys. Rev. B 90, 075404 (2014).

[35] G. D. R. Saito and M. S. Dresselhaus, Physical Properties of Carbon Nanotubes (Imperial College Press, London, 1998).

[36] A. Grüneis, Ph.D. thesis, Tohoku University, 2004.
[37] H. Dumlich, Ph.D. thesis, Freie Universitaet Berlin, 2013.

[38] See Supplemental Material at http://link.aps.org/supplemental/ 10.1103/PhysRevB.97.235417 for additional calculations and derivations, including Refs. [5,6,36,37,41,13,8,42,44,40].

[39] L. E. F. Foa Torres, S. Roche, and J.-C. Charlier, Introduction to Graphene-Based Nanomaterials (Cambridge University Press, Cambridge, 2014).

[40] L. Novotny and B. Hecht, Principles of Nano-Optics, 2nd ed. (Cambridge University Press, Cambridge, 2012).

[41] B. Gu, N. H. Kwong, and R. Binder, Phys. Rev. B 87, 125301 (2013).

[42] R. Binder and N.-H. Kwong, Optical Properties of Graphene (World Scientific, Singapore, 2016), Chap. 1, pp. 3-40.

[43] B. Gallinet, J. Butet, and O. J. F. Martin, Laser Photon. Rev. 9 , 577 (2015).

[44] H. Weyl, Ann. Phys. 365, 481 (1919). 